\title{
A Quasioppositional-Chaotic Symbiotic Organisms Search Algorithm for Distribution Network Reconfiguration with Distributed Generations
}

\author{
Minh-Tuan Nguyen Hoang, ${ }^{1,2}$ Bao-Huy Truong, ${ }^{3}$ Khoa Truong Hoang, ${ }^{4}$ \\ Khanh Dang Tuan, ${ }^{1,2}$ and Dieu Vo Ngoc $\mathbb{B}^{1,2}$ \\ ${ }^{1}$ Department of Power Systems, Ho Chi Minh City University of Technology (HCMUT), 268 Ly Thuong Kiet Street, District 10, \\ Ho Chi Minh City, Vietnam \\ ${ }^{2}$ Vietnam National University Ho Chi Minh, Linh Trung Ward, Thu Duc District, Ho Chi Minh City, Vietnam \\ ${ }^{3}$ Institute of Engineering and Technology, Thu Dau Mot University, Thu Dau Mot City, Binh Duong Province, Vietnam \\ ${ }^{4}$ PEC Technology, 170-170 Bis Bui Thi Xuan Street, Pham Ngu Lao Ward, District 1, Ho Chi Minh City, Vietnam
}

Correspondence should be addressed to Dieu Vo Ngoc; vndieu@hcmut.edu.vn

Received 12 July 2021; Accepted 26 November 2021; Published 24 December 2021

Academic Editor: Trung Thang Nguyen

Copyright (c) 2021 Minh-Tuan Nguyen Hoang et al. This is an open access article distributed under the Creative Commons Attribution License, which permits unrestricted use, distribution, and reproduction in any medium, provided the original work is properly cited.

\begin{abstract}
This study suggests an enhanced metaheuristic method based on the Symbiotic Organisms Search (SOS) algorithm, namely, Quasioppositional Chaotic Symbiotic Organisms Search (QOCSOS). It aims to optimize the network configuration simultaneously and allocate distributed generation (DG) subject to the minimum real power loss in radial distribution networks (RDNs). The suggested method is developed by integrating the Quasiopposition-Based Learning (QOBL) as well as Chaotic Local Search (CLS) approaches into the original SOS algorithm to obtain better global search capacity. The proposed QOCSOS algorithm is tested on 33-, 69-, and 119-bus RDNs to verify its effectiveness. The findings demonstrate that the suggested QOCSOS technique outperformed the original SOS and provided higher-quality alternatives than many other methods studied. Accordingly, the proposed QOCSOS algorithm is favourable in adapting to the DG placement problems and optimal distribution network reconfiguration.
\end{abstract}

\section{Introduction}

In distribution networks, reconfiguration is a traditional technique to minimize power loss in the system by opening/ closing switches to establish the latest optimal network structure. Recently, distributed generation (DG) has been swiftly implemented in distribution networks caused by its great economic, environmental, and technical benefits. The DG unit's optimal allocation in the distribution network can reduce power loss and other risks such as excess reverse power flow, harmonic distortion, overload in line, and overvoltage in the operation of the network [1]. It is realized that the combination of the optimal network reconfiguration and DG placement problems would significantly decrease power loss and enhance the performance of the distribution network. Nevertheless, the distribution network reconfiguration (DNR) is a complex optimization problem since this problem has $2^{n}$ candidate solutions ( $n$ is the number of switches) [2]. Finding the optimal solution among these solutions while satisfying the radial structure and operating constraints is a barrier to problemsolving techniques. In addition, the optimal DG placement (ODGP) problem refers to a complex mixed-integer nonlinear optimization problem. Moreover, this is deemed as an obstacle for optimization methods. Therefore, the issues with ODGP and DNR in combination (DNG-DG) become a more complex optimization problem for the optimization solving approach. This research aims to propose a successful optimization method in solving the DNR-DG problem. 
Merlin and Back initially solved the DNR problem for power loss decrement [3]. In this study, the authors expressed the DNR as a mixed-integer nonlinear optimization problem and overcame it with a discrete branch-andbound method. In [4], Civanlar et al. recommended a branch exchange scheme to address the DNR problem for minimizing balancing load and power loss. In [5], Martín and Gil developed a novel heuristic methodology of branch exchange depending on the branch power flow's direction to decrease the real power loss for the DNR issue. In [6], Gohokar et al. formulated the DNR problem by a network topology concept, in which nodes and branches can be numbered in any order. The single loop optimization procedure was developed to find the optimum network topology. The aforementioned heuristic methods are simple implementation and provide an excellent solution for smallscale problems. Nevertheless, they expose limitations when facing complex optimization problems of changing objective functions. Therefore, these methods have not really attracted researchers.

Advanced optimization methods (i.e., metaheuristics) have been developed and applied to various engineering fields. They are capable of handling various complex constraints and different objective functions. Genetic algorithm (GA) is deemed a well-known metaheuristic, thrivingly implemented on the DNR problem. In [7], a modified GA was suggested for the DNR alongside the power loss reduction objective for 16- and 33-bus RDNs. In [8], GA was enhanced by utilizing the edge-window-decoder encoding system to minimize the power loss via the DNR problem. Another famous metaheuristic approach is particle swarm optimization (PSO). In [9], an adaptive PSO was presented for the RDN's reconfiguration for the real power loss reduction. In [10], the niche binary PSO algorithm was developed to optimally reconfigure the RDN. This algorithm overcame the prematurity of the original PSO for a betterobtained solution. In [11], improved selective binary PSO was offered as an alternative to the DNR to decrease the power loss. Other metaheuristic algorithms have been implemented effectively to the DNR problem, for instance, ant colony search algorithm [12], cuckoo search algorithm (CSA) [13], fireworks algorithm [14], honeybee mating optimization [15], stochastic fractal search [16], and binary group search optimization [17].

The DNR problem becomes more complex when DG units are integrated into distribution networks. Thus, the metaheuristic-based approaches are more suitable than heuristic methods for an optimal solution. Several metaheuristic methods have been recommended for the DNRDG problem. In [2], a harmony search algorithm (HSA) was proposed in solving the DNR-DG problem for the 33- and 69-bus RDNs. The aim of this research was voltage profile increment and real power loss reduction. Also, in studies $[18,19]$, fireworks algorithm (FWA) and adaptive cuckoo search algorithm (ACSA) were, respectively, presented for the DNR-DG within the similar coverage in the research [2]. In [20], the DNR-DG problem was fixed by the adaptive shuffled frogs leaping algorithm (ASFLA). In this study, the simulated outcomes from 33-bus and 69-bus RDNs for various circumstances found that the ASFLA was efficient than ACSA, FWA, and SFLA. In [21], the salp swarm algorithm (SSA) was suggested for handling the DNR problem with the DG placement. The effectiveness of SSA was also tested on 33- and 69-bus RDNs. Generally, the aforementioned metaheuristic methods were implemented in smalland medium-scale test systems. The research did not take into account large-scale systems. Moreover, the majority of the research employed metaheuristic approaches to tackle the DNR-DG issue. Despite these techniques having a significant search capacity for an optimal solution, there is no guarantee that they would be effective for all optimization problems. A metaheuristic method can effectively solve a specific optimization problem; however, it may not be effective for others. Therefore, there is always room to suggest new effective metaheuristic methods for dealing with complex optimization problems.

This research suggests a powerful optimization strategy to manage the DNR-DG problem towards minimum real power loss. The suggested approach is the Quasioppositional Chaotic Symbiotic Organisms Search (QOCSOS) method developed in our previous work [22]. The QOCSOS algorithm embedded QOBL and CLS search strategies to boost the obtained solution quality and convergence speed of the original SOS. In QOCSOS, the QOBL strategy helps the algorithm to explore more promising domains; thus, it increases the chance of obtaining a better solution. As a result, the algorithm's exploration capacity is enhanced. In addition to QOBL, the CLS strategy also helps the algorithm to avoid trapping in local optima. It locally explores the neighbourhood of the current best solution for better exploitation. Consequently, the integration of both QOBL and CLS strategies would keep a balance between exploration and exploitation and significantly improve the performance of the SOS algorithm. The suggested QOCSOS technique is applied to simultaneously obtain the optimal configuration and DG placement in the 33-, 69-, and 119-bus RDNs.

This research's contributions are outlined as the statements as follows:

(i) The QOCSOS was modified to the DNR-DG problem for power loss decrement.

(ii) The QOCSOS was successfully applied to 119-bus large-scale system for the DNR-DG problem.

(iii) The simulation results showed that the simultaneous consideration of optimal network reconfiguration and DG placement substantially enhanced the distribution networks' performance with regard to voltage profile and power loss compared to only network reconfiguration or DG placement.

(iv) The outcome comparison illustrated that the QOCSOS technique is more successful than the original SOS and other compared approaches for the acquired excellent solution standard.

The remaining sections of the paper are as follows. Section 2 describes the problem formulation of the DNRDG. Section 3 presents the QOCSOS algorithm, in which the original SOS, QOBL, and CLS are introduced. Section 4 
explains the implementation of the proposed QOCSOS to the DNR-DG problem. The results of numerical simulations are presented in Section 5. Finally, the conclusions are given in Section 6.

\section{Problem Formulation}

The main goal of the DNR-DG problem is to minimize real power loss $\left(P_{L}\right)$ in RDNs, while all operating constraints are satisfied as follows:

$$
\begin{aligned}
O F & =\operatorname{Min}(P)_{L} \\
& =\operatorname{Min}\left(\sum_{k=1}^{N_{L}} R_{k} I_{k}^{2}\right),
\end{aligned}
$$

where $R_{k}$ is the resistance of the $k^{\text {th }}$ branch, $I_{k}$ is the current passing through that branch, and $N_{L}$ is the number of branches in an RDN.

The operational constraints for the objective function in equation (1) are given as follows:

i. Power balance constraints:

$$
\begin{gathered}
P_{S S}+\sum_{i=1}^{N_{D G}} P_{D G, i}=\sum_{j=1}^{N_{B}} P_{D, j}+\sum_{k=1}^{N_{L}} P_{L, k}, \\
Q_{S S}+\sum_{i=1}^{N_{D G}} Q_{D G, i}=\sum_{j=1}^{N_{B}} Q_{D, j}+\sum_{k=1}^{N_{L}} Q_{L, k},
\end{gathered}
$$

where $N_{D G}$ is the number of DGs; $N_{B}$ is the number of buses in $\mathrm{RDN} ; P_{D, j}$ and $Q_{D, j}$ are the active and reactive power of load demands at the $j^{\text {th }}$ bus, respectively; $P_{L, k}$ and $Q_{L, k}$ are the active and reactive power losses in the $k^{\text {th }}$ branch, respectively; $P_{S S}$ and $Q_{S S}$ are the active and reactive power outputs at the slack bus, respectively; $P_{D G, i}$ and $Q_{D G, i}$ are the active and reactive power outputs of the $i^{\text {th }}$ DG, respectively.

ii. Voltage constraint:

$$
V_{\min , i} \leq V_{i} \leq V_{\max , i} ; \quad i=1, \ldots, N_{B},
$$

where $V_{\min , i}$ and $V_{\max , i}$ denote the voltage bounds at the $i^{\text {th }}$ bus.

iii. Thermal limit:

$$
\left|I_{k}\right| \leq\left|I_{\max , k}\right| ; \quad k=1, \ldots, N_{L},
$$

where $I_{\max , k}$ represents the maximum current allowed to flow through the $k^{\text {th }}$ branch.

iv DG generation constraint:

$$
P_{D G \min , i} \leq P_{D G, i} \leq P_{D G \max , i} ; \quad i=1, \ldots, N_{D G},
$$

where $P_{D G \min , i}$ and $P_{D G \max , i}$ denote the capacity limit of the $i^{\text {th }} \mathrm{DG}$, respectively.

v DG penetration constraint:

$$
\sum_{i=1}^{N_{D G}} P_{D G, i} \leq \sum_{j=1}^{N_{B}} P_{D, j} .
$$

vi Radial configuration constraint:

The radial topology must be maintained after reconfiguration as follows [23]:

$$
\operatorname{det}(A)=1 \text { or }-1 \text {, }
$$

where $A$ represents a matrix for the connection of branches and buses in the RDN [23]:

$$
A_{i j}= \begin{cases}1, & \text { if branch } i \text { connects from bus } j, \\ -1, & \text { if branch } i \text { connects to bus } j, \\ 0, & \text { otherwise. }\end{cases}
$$

\section{QOCSOS Algorithm}

3.1. Original SOS Algorithm. The SOS method was developed based on a natural ecosystem with symbiotic relations between two different organisms [24]. The search process is started by randomly generating a population of organisms (ecosystem) as follows:

$$
O_{i}=O_{i \min }+\operatorname{rand}(0,1) \times\left(O_{i \max }-O_{i \min }\right), \quad i=1,2, \&, N,
$$

where $O_{i}=\left[o_{i 1}, o_{i 2}, \ldots, o_{i D}\right] ; D$ is the dimension of optimization problem; $O_{i \min }$ and $O_{i \max }$ are the upper and lower limits of the $i^{\text {th }}$ organism, respectively; $N$ is the size of the ecosystem.

In the population, each organism represents a solution. For each iteration, the population is updated based on mutualism, commensalism, and parasitism phases. Each phase is defined as follows.

3.1.1. Mutualism. Based on mutualistic relationships, the $j^{\text {th }}$ random organism is assigned from the population to associate with the $i^{\text {th }}$ organism during this phase. Vectors $O_{i}$ and $O_{j}$ are the $i^{\text {th }}$ and $j^{\text {th }}$ organisms in the population, respectively. New organisms are created as follows [24]:

$$
\begin{aligned}
O_{i}^{\text {new }} & =O_{i}+\operatorname{rand}(0,1) \times\left(O_{\text {best }}-M V \times b f_{1}\right), \\
O_{j}^{\text {new }} & \left.=O_{j}+\operatorname{rand}(0,1) \times O_{\text {best }}-M V \times b f_{2}\right), \\
M V & =\frac{O_{i}+O_{j}}{2},
\end{aligned}
$$

where $M V$ is the average of the $i^{\text {th }}$ and $j^{\text {th }}$ organisms, representing a mutualistic relationship; $b f_{1}$ and $b f_{2}$ are randomly chosen as 1 or $2 ; O_{\text {best }}$ denotes the best organism in the population.

The fitness value is calculated for each organism. The new organism is updated as follows: 


$$
O_{i}= \begin{cases}O_{i}^{\text {new }}, & \text { if } f\left(O_{i}^{\text {new }}\right)<f\left(O_{i}\right), \\ O_{i}, & O_{i} \text { otherwise. }\end{cases}
$$

3.1.2. Commensalism. In this phase, the $i^{\text {th }}$ organism interacts with the $j^{\text {th }}$ organism, which is randomly selected from the population based on commensalism interaction. A new organism is generated as follows [24]:

$$
O_{i}^{\text {new }}=O_{i}+\operatorname{rand}(-1,1) \times\left(O_{\text {best }}-O_{j}\right) .
$$

The new organism is updated as described by equation (10).

3.1.3. Parasitism. In this phase, the $i^{\text {th }}$ organism acts as a parasite, and the $j^{\text {th }}$ random organism acts as the host. In the parasitism interaction of two different organisms, the parasite gets benefits, while the host gets harm. Vector $O_{i}$ is duplicated to create a Parasite_Vector $(P V)$. A new candidate solution $\left(O_{P V}\right)$ is created by randomly modifying some variables of the $P V$ vector [24]. The $O_{P V}$ vector is updated or discarded as follows:

$$
O_{j}= \begin{cases}O_{P V}, & \text { if } f\left(O_{P V}\right)<f\left(O_{j}\right), \\ O_{j}, & O_{i} \text { otherwise. }\end{cases}
$$

3.2. QOBL Strategy. The QOBL strategy is performed when SOS generates a new population of organisms. The QOBL approach is also applied when the initial population is randomly initialized. The opposite point $O_{i}^{o}$ of each organism $O_{i}$ is calculated as follows [25]:

$$
O_{i}^{o}=O_{i \min }+O_{i \max }-O_{i}
$$

Then, the quasiopposite point $O_{i}^{q o}$ is given by the following equation [22]:

$$
O_{i}^{q o}=\operatorname{rand}\left(\frac{O_{i \min }+O_{i \max }}{2}, O_{i}^{o}\right) \text {. }
$$

The pseudocode of QOBL (Algorithm 1) is illustrated in Figure 1.

3.3. CLS Strategy. For increasing the likelihood of finding better solutions, the CLS approach is utilized to explore the vicinity of the current best solution. A new candidate solution is created via the CLS strategy as follows [27]:

$$
O_{\text {best }, k}^{\text {new }}=O_{\text {best }, k}+\left(Z_{k}-0.5\right) \times\left(O_{i, k}-O_{j, k}\right),
$$

where $O_{\text {best }, k}^{\text {new }}$ is the new organism created via CLS at the $k^{\text {th }}$ iteration; $O_{b e s t, k}$ is the current best organism; $O_{i, k}$ and $O_{j, k}$ denote the two random organisms selected from the population, respectively; $Z_{k}$ is generated from "logistic map" [28].

The fitness values are computed for the organisms $O_{\text {best }, k}^{\text {new }}$ and $O_{b e s t, k}$. The new organism is updated as follows:

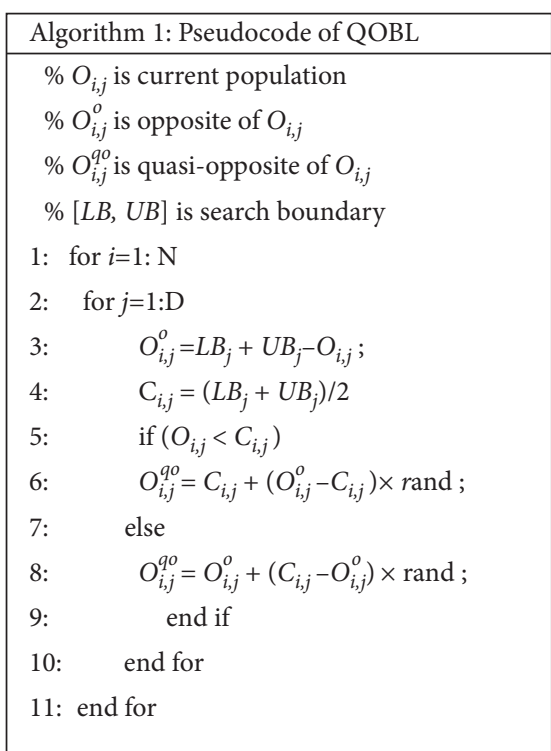

Figure 1: QOBL pseudocode.

$$
O_{\text {best }, k}= \begin{cases}O_{\text {best }, k}^{\text {new }}, & \text { if } f\left(O_{\text {best }, k}^{\text {new }}\right)<f\left(O_{\text {best }, k}\right), \\ O_{\text {best }, k}, & \text { otherwise. }\end{cases}
$$

3.4. QOCSOS. The QOCSOS method is developed based on the original SOS with the integration of QOBL and CLS strategies. Firstly, QOCSOS generates a population of organisms $\mathrm{O}$. Afterwards, the quasiopposite population $\mathrm{O}^{q o}$ is created via the QOBL strategy. From the set $\left[O, O^{q o}\right]$, QOCSOS selects $N$ (i.e., size of the ecosystem) of best organisms as an initial population according to their best fitness values. Next, the operation of SOS is performed. At the end of this stage, when a new population is created, a jumping rate $j_{r}$ parameter determines whether to implement the QOBL approach or to keep the current population. Lastly, the CLS approach is used to obtain the best organism. The QOCSOS operation is implemented until the stopping condition is satisfied. Figure 2 shows the pseudocode of the QOCSOS method.

\section{Implementation of QOCSOS to DNR- DG Problem}

This section discussed how QOCSOS was deployed to the DNR-DG issue to minimize real power loss in RDNs. Therefore, three scenarios are considered as follows:

(i) Case 1: considering only the DNR.

(ii) Case 2: considering only the optimal DG location.

(iii) Case 3: simultaneous consideration of DNR and optimal DG location (DNR-DG problem).

4.1. Initialization of Population. In the population of QOCSOS, each organism $O_{i}(i=1, \ldots, N)$ denotes a solution vector, which consists of opened switches, locations, and 


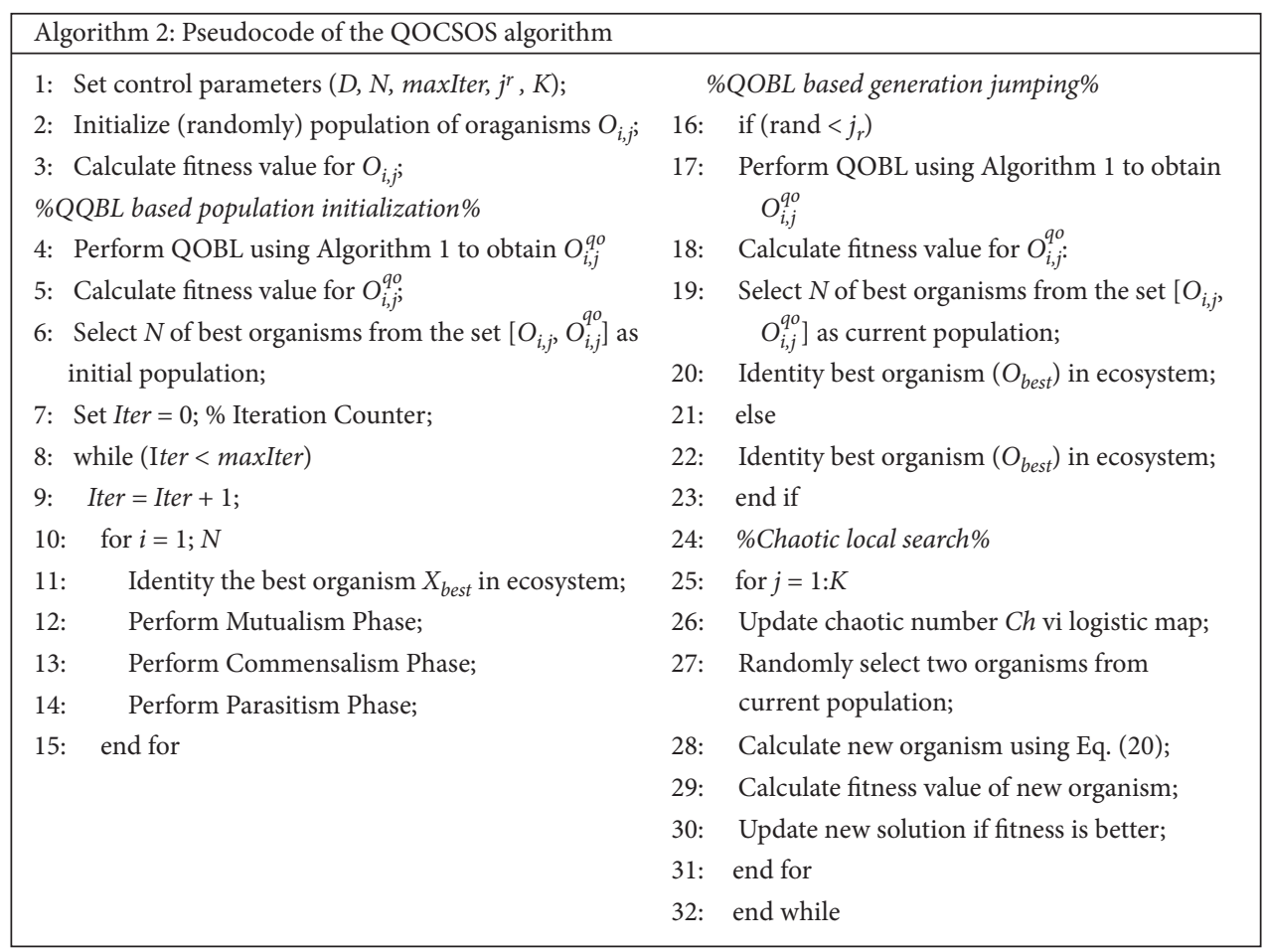

FIGURE 2: Pseudocode of the QOCSOS algorithm.

capacities of DGs. Equations (18), (19), and (20) express a solution for Cases 1, 2, and 3, respectively.

$$
\begin{aligned}
O_{i} & =\left[S W_{1}, \ldots, S W_{N_{S W}}\right] \\
O_{i} & =\left[L_{D G, 1}, \ldots, L_{D G, N_{D G}}, P_{D G, 1}, \ldots, P_{D G, N_{D G}}\right] \\
O_{i} & =\left[S W_{1}, \ldots, S W_{N_{S W}}, L_{D G, 1}, \ldots, L_{D G, N_{D G}}, P_{D G, 1}, \ldots, P_{D G, N_{D G}}\right],
\end{aligned}
$$

in which $N_{S W}$ denotes the number of opened switches.

Each organism is randomly generated in its boundaries, in which the opened switches and locations of DGs are natural numbers. Hence, the designed variables are generated as follows:

$$
\begin{aligned}
S W_{i} & =\operatorname{round}\left[S W_{\min , i}+\operatorname{rand}(0,1) \times\left(S W_{\max , i}-S W_{\min , i}\right)\right], \\
L_{D G, j} & =\operatorname{round}\left[L_{D G \min , j}+\operatorname{rand}(0,1) \times\left(L_{D G \max , j}-L_{D G \min , j}\right],\right. \\
P_{D G, j} & =P_{D G \min , j}+\operatorname{rand}(0,1) \times\left(P_{D G \max , j}-P_{D G \min , j}\right),
\end{aligned}
$$

in which $i=1,2, \ldots, N_{S W} ; j=1,2, \ldots, N_{D G} ; S W_{\min , i}=1$ for all variables; $S W_{\max , i}$ denotes the length of the $i^{\text {th }}$ fundamental loop vectors. The principle of finding the fundamental loop vectors can be found in [29]; $L_{D G \min , j}=2$ for all variables, which means that DG units can be installed at all buses except the slack bus.

4.2. Fitness Value. Fitness values for organisms of QOCSOS are calculated as follows:

$$
F_{T}=O F+K_{p} \sum_{i=1}^{N_{B}}\left(V_{i}-V_{i}^{\lim }\right)^{2}+K_{q} \sum_{k=1}^{N_{L}}\left(I_{k}-I_{k}^{\lim }\right)^{2}
$$

in which $K_{p}$ and $K_{q}$ denote penalty coefficients for voltage and thermal current, respectively; $x^{\lim }$ denotes the limit value of the dependent variable $x$ (bus voltages and currents) as follows:

$$
x^{\lim }= \begin{cases}x_{\max }, & \text { if } x>x_{\max } \\ x_{\min }, & \text { if } x<x_{\min } \\ x, & \text { otherwise }\end{cases}
$$

where $x$ denotes the $V_{i}$ and $I_{k}$ values; $x^{\text {lim }}$ denotes the limitations of $V_{i}$ and $I_{k}$.

4.3. Overall Procedure. The QOCSOS implementation to the DNR-DG problem can be drawn as follows:

Step 1. set QOCSOS parameters $\left(D, N\right.$, maxIter, $\left.j_{r}, K\right)$.

Step 2. perform the fundamental loops to define the lower and upper bounds of $S W_{i}$

Step 3. randomly create a population of organisms $O_{i, j}$. Execute the QOBL approach to acquire $O_{i, j}^{q 0}$. Calculate fitness values using equation (22) for the set $\left[O i, O_{i, j}^{q o}\right]$. Choose $N$ of best organisms from the set $\left[\mathrm{Oi}, \mathrm{O}_{i, j}^{q o}\right]$ as initial population. Check the radial structure for all organisms. Set Iter $=0$.

Step 4. set Iter $=$ Iter +1 . Start the main loop. 
Step 5. define the best organism $\mathrm{O}_{\text {best }}$ having the best fitness value.

Step 6. execute the mutualism phase.

Step 7. execute the commensalism phase.

Step 8. execute the parasitism phase.

(In each phase, check the radial condition and constraints for new organisms and apply an approach using equation (23) if any organism violates its limits.)

Step 9. move to Step 5 if organism $O_{\mathrm{i}}$ is not the final organism of the ecosystem $\left(O_{\mathrm{N}}\right)$. Otherwise, continue to the next step. Check the radial constraint and apply the repairing strategy if necessary.

Step 10. if $\operatorname{rand}()<j_{\mathrm{r}}$, implement the QOBL approach to obtain quasiopposite points of the current population. Check the radial constraint and deploy the repairing strategy. Calculate fitness value and define the best organism $\mathrm{O}_{\text {best }}$. Otherwise, define the best organism $\mathrm{O}_{\text {best }}$ from Step 9.

Step 11. implement the CLS approach to obtain the best organism $\mathrm{O}_{\text {best }}$. Check the radial constraint and apply the repairing strategy

Step 12. if Iter $<$ maxIter, go to Step 4. Else, the process is done.

\section{Simulation Results}

In this study, 33-bus, 69-bus, and 118-bus RDNs were used to test the QOCSOS method with three scenarios. The QOCSOS technique was performed in thirty separate trials for each case study to find the optimum solution. Besides, the SOS approach was also applied to the same problem for result comparisons. QOCSOS's initial parameters were chosen in Table 1.

5.1. 33-Bus Test Network. The proposed QOCSOS method was initially implemented on the 33-bus RDN. The comprehensive data of this network was obtained from [30]. For Case 1, the QOCSOS method obtained the opened switches: 7-9-14-32-37, where the real power loss of the network was decreased from $202.67 \mathrm{~kW}$ (base case) to $139.5513 \mathrm{~kW}$ in relation to $31.14 \%$ of the power loss reduction (PLR). The system's minimum voltage amplitude was raised from 0.9131 p.u. to 0.9378 p.u. Table 2 shows the reconfiguration results acquired using various optimization methods for the 33-bus RDN. For accurate comparison of the best obtained result improvement (power loss), the following equation can be used to assess quantitatively the result improvement:

$$
R I_{\mathrm{QOCSOS}} \%=\frac{R_{\text {compared_method }}-R_{\mathrm{QOCSOS}}}{R_{\text {compared_method }}} \times 100 \%,
$$

where $R I_{\mathrm{QOCSOS}}$ is the QOCSOS result improvement compared to other methods; $R_{\text {compared_method }}$ is the power loss obtained by other compared methods; $R_{\mathrm{QOCSOS}}$ is the power loss obtained by the QOCSOS method.

In equation (24), the best result improvement $(R I)$ can be the plus sign (+) or minus sign (-). If RI has a plus sign (+), the result obtained by QOCSOS is better than the compared method; otherwise, the compared method has a better result than QOCSOS. Besides, if RI is zero, QOCSOS and compared methods have the same result.

From Table 2, the RI values show that the real power loss acquired by the QOCSOS was slightly better than FWA [14], ACSA [19], HSA [31], and ITS [31] (the result improvement is from $0.3079 \%$ to $2.0211 \%$ ). The methods including SOS, ICSA [13], RGA [7], SSA [21], and SFS [16] have similar power loss compared to the proposed QOCSOS.

For Case 2, where three DG units were installed in the network, the QOCSOS determined the optimum positions to place the DG units at buses 14,24 , and 30 with capacities of $0.7540 \mathrm{MW}, 1.0994 \mathrm{MW}$, and 1.0714 MW. Consequently, a minimal real power loss of $71.4572 \mathrm{~kW}(64.74 \%$ PLR) and a minimal voltage of 0.9687 p.u. were established. Table 3 portrays the findings of optimal DG placement obtained by QOCSOS and distinct methods for the 33-bus RDN. It can be displayed that QOCSOS had the same power loss as SSA [21] and was lower than those of other methods in the table. The observation of RI shows the proposed QOCSOS method can improve the result of $0.0179 \%, 26.1501 \%, 19.4213 \%$, and $3.7743 \%$ compared to SFS [16], HSA [2], FWA [14], and ACSA [19], respectively.

For Case 3, QOCSOS acquired the best network configuration using the opened switches: 10-28-31-33-34. Concurrently, DGs were deployed to positions at buses 7, 18, and 25 having capacities of $0.8708 \mathrm{MW}, 0.7118 \mathrm{MW}$, and 1.2274 MW. After optimal DNR-DG problem, the minimum real power loss for this case obtained by the QOCSOS was $51.5388 \mathrm{~kW}$, corresponding to a PLR of $74.57 \%$. Table 4 displays the findings of DNR-DG obtained by QOCSOS and different methods for the 33-bus RDN. Based on the RI values with the plus sign "+," the QOCSOS method obtained the best real power loss results among the other compared methods for this case.

Tables 2-4 show that the PLR percentages for Cases 1-3 were $31.14 \%, 64.74 \%$, and $74.57 \%$, accordingly. The PLR of Case 3 was the largest of the three instances. This demonstrated that considering DNR and ODGP at the same time has a substantial influence on power loss reduction. The convergence curves of the QOCSOS and the original SOS are shown in Figure 3. For Cases 1 and 2, both the SOS and QOCSOS algorithms yielded the equivalent real power loss outcome. Nevertheless, the QOCSOS's convergence speeds for optimal outcomes were faster than SOS in all scenarios. Moreover, the voltage profiles of the 33-bus test network for Cases 1,2, and 3 are illustrated in Figure 4. When the scenario of simultaneous consideration of ODGP and DNR (Case 3) was addressed, the network's voltage profile was greatly enhanced. 
TABLE 1: Setting parameters of QOCSOS and SOS for three test networks.

\begin{tabular}{lcc}
\hline 33-bus RDN & 69-bus RDN & 118 -bus RDN \\
\hline$N=20 ;$ maxIter $=50$ & $N=20 ;$ maxIter $=100$ & $N=100$ and maxIter $=100$ for case 1 and case $2 ; N=100$ and maxIter $=200$ for case 3 \\
\hline
\end{tabular}

TABLE 2: Optimal configuration obtained by QOCSOS and other techniques for Case 1 of the 33-bus RDN.

\begin{tabular}{lccccc}
\hline Method & Opened switches & $P_{L}(\mathrm{~kW})$ & $R I(\%)$ & $P L R(\%)$ & - \\
\hline Base case & $33-34-35-36-37$ & 202.68 & & 31.15 & 0.9131 \\
RGA [7] & $7-9-14-32-37$ & 139.55 & -0.0009 & 29.86 & 0.9378 \\
ITS [31] & $7-9-14-36-37$ & 142.17 & +1.8419 & 29.73 & 0.9336 \\
HSA [31] & $7-10-14-28-36$ & 142.43 & +2.0211 & 30.94 & 0.9377 \\
ACSA [19] & $7-9-14-28-32$ & 139.9823 & +0.3079 & 30.94 & 0.9412 \\
FWA [14] & $7-9-14-28-32$ & 139.9823 & +0.3079 & 31.14 & 0.9412 \\
ICSA [13] & $7-9-14-32-37$ & 139.55 & -0.0009 & 31.14 & 0.9378 \\
SSA [21] & $7-9-14-32-37$ & 139.55 & -0.0009 & 31.14 & 0.9378 \\
SFS [16] & $7-9-14-32-37$ & 139.55 & -0.0009 & 31.14 & 0.9378 \\
SOS & $7-9-14-32-37$ & 139.5513 & 0.0000 & 0.9378 \\
QOCSOS & $7-9-14-32-37$ & 139.5513 & & & \\
\hline
\end{tabular}

5.2. 69-Bus Test Network. The QOCSOS technique was used on the 69-bus RDN to verify its scalability. The data are presented and tabulated in [32]. Table 5 portrays the findings of the DNR problem obtained by QOCSOS and other techniques for the 69-bus RDN. For Case 1, the switches, 1457-61-69-70, were opened by the QOCSOS to form the system's optimal configuration. Then, the system's real power loss was $98.6062 \mathrm{~kW}$, which corresponds to a PLR of $56.17 \%$ in comparison to the baseline case. According to the $R I$ values, the real power loss obtained by QOCSOS is slightly better than SFS [16], SSA [21], and HSA [2] and close to that of other methods, as seen in Table 5 .

Table 6 presents the results of the ODGP problem obtained by QOCSOS, SOS, ACSA [19], FWA [14], HSA [2], SSA [21], and SFS [16]. For Case 2, QOCSOS found three buses, 11, 18, and 61, to install three DGs. The corresponding DGs capacities were $0.5268 \mathrm{MW}, 0.3804 \mathrm{MW}$, and $1.7190 \mathrm{MW}$. The system's real power loss is reduced from $225.0005 \mathrm{~kW}$ (base case) to $69.4284 \mathrm{~kW}$ with the DG installation, which is equal to SOS, close to SSA [21], and better than other methods.

Table 7 reports the results of the DNR-DG problem yielded by QOCSOS and other algorithms for the 69-bus network. QOCSOS simultaneously obtained the optimal opened switches: 14-58-61-69-70. Moreover, three DGs of capacities $0.5376 \mathrm{MW}, 1.4340 \mathrm{MW}$, and $0.4903 \mathrm{MW}$ were located at buses 11,61 , and 164, correspondingly. As a result, the obtained real power loss was $35.1624 \mathrm{~kW}$, which is the same as SOS and SFS [16] and better than other compared methods.

From Tables 4-7, the QOCSOS generated the real power losses of $98.6062 \mathrm{~kW}, 69.4284 \mathrm{~kW}$, and $35.1624 \mathrm{~kW}$ for the cases of 1 to 3 , accordingly. In comparison to Cases 1 and 2, the real power loss from Case 3 was the smallest. This demonstrated that simultaneously considering ODGP and DNR problems greatly decreased the system's real power loss. Figure 5 shows the QOCSOS and SOS's convergence characteristics for Cases 1-3. In every case, QOCSOS outperformed SOS in terms of convergence speed. The network's minimal voltage value was 0.9495 p.u., 0.9790 p.u., and 0.9813 p.u. for Cases 1-3, accordingly, after optimization for three cases. These reduced voltage values were enhanced from 0.9092 p.u. (base case), indicating that the network's voltage profile has improved significantly (Figure 6). Case 3 had the greatest minimum voltage out of the three cases. This indicated that taking into account both DNR and ODGP concurrently boosted the system's voltage profile tremendously.

5.3. 119-Bus Test Network. The effectiveness of the QOCSOS technique was justified on the large-scale 119-bus RDN. The data for branch and network load was provided in [33]. Table 8 demonstrates the outcomes of QOCSOS and other techniques for the DNR problem of the 119-bus RDN. As observed from this table, QOCSOS acquired the opened switches, 23-25-34-39-42-50-58-71-74-95-97109-121-129-130, generating a real power loss of $854.0309 \mathrm{~kW}$. It can be from $R I$ values that this result was slightly better than that of SFS [16] and better than other compared methods.

Table 9 presents the results offered by QOCSOS and other methods for the ODGP problem. For this case, QOCSOS found that three DGs were located at buses 50, 71, and 109, along with the corresponding capacities of 2.8833 MW, 2.9785 MW, and 3.1199 MW. The power loss result acquired by QOCSOS was identical to those of SOS and SFS [16] methods.

Table 10 tabulates the results gathered by QOCSOS and various approaches for the DNR-DG problem. For this case, QOCSOS obtained the opened switches: 23-26-34-39-42-5061-70-74-85-86-107-121-125-129, where three DGs were located at buses 4,91 , and 110 with capacities of $9.3909 \mathrm{MW}$, 4.1078 MW, and 2.5599 MW, accordingly. When both ODGP and DNR were implemented at the same time, the obtained real power loss of QOCSOS was $565.0605 \mathrm{~kW}$, 
TABLE 3: Optimal DG placement results obtained by QOCSOS and other techniques for Case 2 of 33-bus RDN.

\begin{tabular}{|c|c|c|c|c|c|}
\hline Method & $P_{D G}(\mathrm{MW}) /$ (bus) & $P_{L}(\mathrm{~kW})$ & $R I(\%)$ & PLR (\%) & $V_{\min }$ (p.u.) \\
\hline ACSA [19] & $0.7798 /(14) ; 1.1251 /(24) ; 1.3496 /(30)$ & 74.26 & +3.7743 & 63.26 & 0.9778 \\
\hline FWA [14] & $0.5897 /(14) ; 0.1895 /(18) ; 1.0146 /(32)$ & 88.68 & +19.4213 & 56.24 & 0.9680 \\
\hline HSA [2] & $0.1070 /(18) ; 0.5724 /(17) ; 1.0462 /(33)$ & 96.76 & +26.1501 & 52.26 & 0.9670 \\
\hline SSA $[21]$ & - & 71.45 & -0.0101 & 64.74 & 0.9687 \\
\hline SFS [16] & $0.7540 /(14) ; 1.0994 /(24) ; 1.0714 /(30)$ & 71.47 & +0.0179 & 64.74 & 0.9687 \\
\hline SOS & $0.7539 /(14) ; 1.0994 /(24) ; 1.0717 /(30)$ & 71.4572 & 0.0000 & 64.74 & 0.9687 \\
\hline QOCSOS & $0.7540 /(14) ; 1.0994 /(24) ; 1.0714 /(30)$ & 71.4572 & & 64.74 & 0.9687 \\
\hline
\end{tabular}

TABLE 4: Optimal DRN-DG problem results obtained by QOCSOS and other techniques for Case 3 of 33-bus RDN.

\begin{tabular}{|c|c|c|c|c|c|c|}
\hline Method & Opened switches & $P_{D G}(\mathrm{MW}) /$ (bus) & $P_{L}(\mathrm{~kW})$ & $R I(\%)$ & PLR (\%) & $V_{\min }$ (p.u.) \\
\hline ACSA [19] & $33-34-11-31-28$ & $0.8968 /(18) ; 1.4381 /(25) ; 0.9646 /(7)$ & 53.21 & +3.1408 & 73.75 & 0.9806 \\
\hline SSA [21] & $6-14-11-17-28$ & - & 56.42 & +8.6515 & 72.16 & 0.9762 \\
\hline SFS [16] & $7-9-14-27-30$ & $0.7753 /(22) ; 0.7356 /(33) ; 1.2858 /(25)$ & 53.01 & +2.7753 & 73.85 & 0.9720 \\
\hline SOS & $6-34-11-31-28$ & $0.7082 /(17) ; 1.4072 /(25) ; 0.7051 /(8)$ & 53.0662 & +2.8783 & 73.82 & 0.9690 \\
\hline QOCSOS & $10-28-31-33-34$ & $0.8708 /(7) ; 0.7118 /(18) ; 1.2274 /(25)$ & 51.5388 & & 74.57 & 0.9719 \\
\hline
\end{tabular}

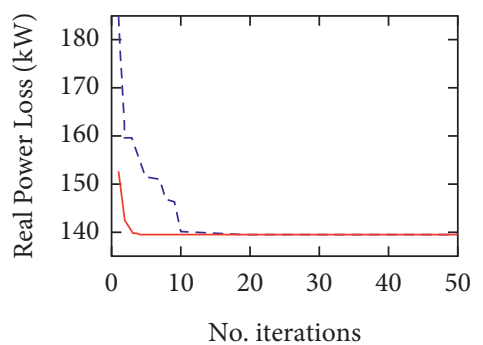

Case 1

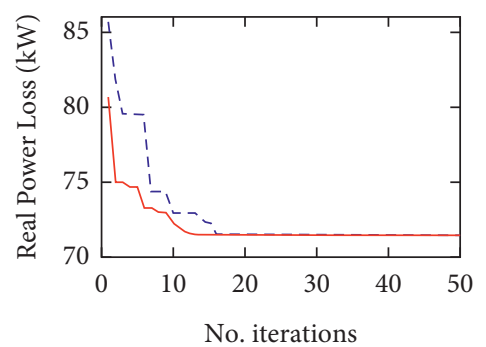

Case 2

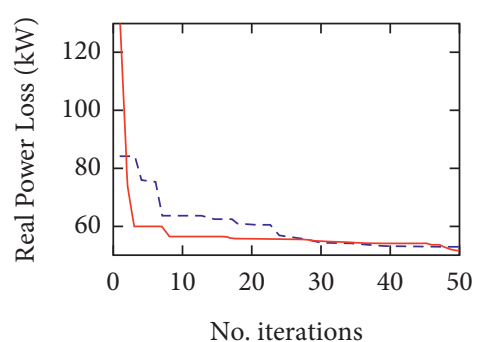

Case 3
$---\operatorname{SOS}$
$---\operatorname{SOS}$
$---\operatorname{SOS}$
- QOCSOS
— QOCSOS
— QOCSOS

FIgure 3: Convergence curves by QOCSOS and SOS for all cases of 33-bus network. (a) Case 1. (b) Case 2. (c) Case 3.

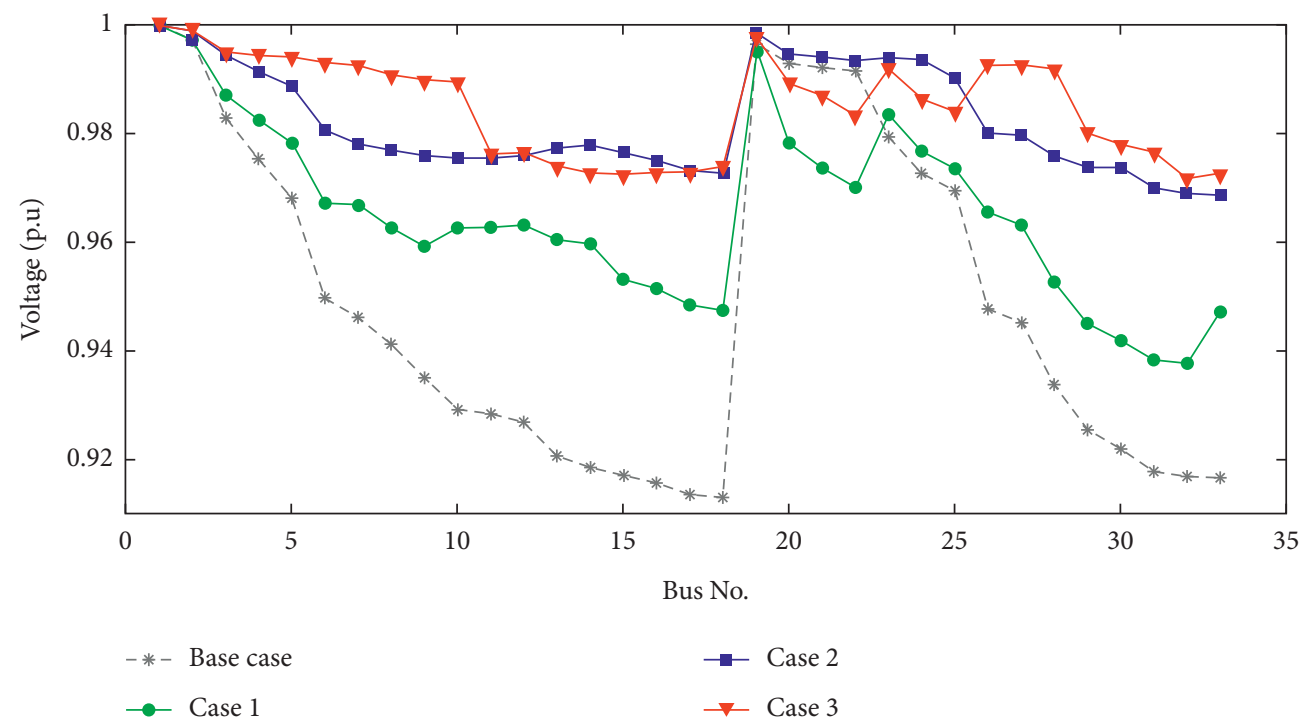

FIGURE 4: Voltage profile for all cases for 33-bus network. 
TABLE 5: Optimal configuration obtained by QOCSOS and other techniques for Case 1 of the 69-bus RDN.

\begin{tabular}{|c|c|c|c|c|c|}
\hline Method & Opened switches & $P_{L}(\mathrm{~kW})$ & $R I(\%)$ & PLR (\%) & $V_{\min }$ (p.u.) \\
\hline Base case & $69-70-71-72-73$ & 225.0005 & & - & 0.9092 \\
\hline ASFLA [20] & $69-71-14-57-61$ & 98.59 & -0.0164 & 56.16 & 0.9495 \\
\hline HSA [2] & $69-18-13-56-61$ & 99.35 & +0.7487 & 55.85 & 0.9428 \\
\hline ACSA [19] & $69-70-14-57-61$ & 98.59 & -0.0164 & 56.16 & 0.9495 \\
\hline FWA [14] & $69-70-14-56-61$ & 98.56 & -0.0469 & 56.16 & 0.9495 \\
\hline SSA [21] & $69-14-71-61-58$ & 98.63 & +0.0241 & 56.15 & 0.9492 \\
\hline SFS [16] & $14-55-61-69-70$ & 98.62 & +0.0140 & 56.17 & 0.9495 \\
\hline SOS & $14-57-61-69-70$ & 98.6062 & 0.0000 & 56.17 & 0.9495 \\
\hline QOCSOS & $14-57-61-69-70$ & 98.6062 & & 56.17 & 0.9495 \\
\hline
\end{tabular}

TABLe 6: Optimal DG placement results obtained by QOCSOS and other techniques of Case 2 of 33-bus RDN.

\begin{tabular}{|c|c|c|c|c|c|}
\hline Method & $P_{D G}(\mathrm{MW}) /($ bus $)$ & $P_{L}(\mathrm{~kW})$ & $R I(\%)$ & PLR (\%) & $V_{\min }$ (p.u.) \\
\hline ACSA [19] & $0.6022 /(11) ; 0.3804 /(18) ; 2.0000 /(61)$ & 72.44 & +4.1574 & 67.79 & 0.9890 \\
\hline FWA [14] & $0.4085 /(65) ; 1.1986 /(61) ; 0.2258 /(27)$ & 77.85 & +10.8177 & 65.39 & 0.9740 \\
\hline HSA [2] & $0.1018 /(65) ; 0.3690 /(64) ; 1.3024 /(63)$ & 86.77 & +19.9857 & 61.43 & 0.9677 \\
\hline SSA [21] & - & 69.41 & -0.0265 & 69.14 & 0.9789 \\
\hline SFS [16] & $0.5268 /(11) ; 0.3804 /(18) ; 1.7190 /(61)$ & 69.44 & +0.0167 & 69.14 & 0.9790 \\
\hline SOS & $0.5268 /(11) ; 0.3804 /(18) ; 1.7190 /(61)$ & 69.4284 & 0.0000 & 64.74 & 0.9687 \\
\hline QOCSOS & $0.5268 /(11) ; 0.3804 /(18) ; 1.7190 /(61)$ & 69.4284 & & 69.14 & 0.9790 \\
\hline
\end{tabular}

TABLE 7: Optimal DRN-DG problem results obtained by QOCSOS and other techniques of Case 3 for 69-bus RDN.

\begin{tabular}{|c|c|c|c|c|c|c|}
\hline Method & Opened switches & $P_{D G}(\mathrm{MW}) /($ bus $)$ & $P_{L}(\mathrm{~kW})$ & $R I(\%)$ & $P L R(\%)$ & $V_{\min }$ (p.u.) \\
\hline ACSA [19] & $69-70-14-58-61$ & $0.5413 /(11) ; 0.5536 /(65) ; 1.7240 /(61)$ & 37.02 & +5.0178 & 83.54 & 0.9869 \\
\hline SSA [21] & $69-14-70-63-58$ & - & 35.81 & -1.8084 & 84.08 & 0.9808 \\
\hline SFS [16] & $14-56-61-69-70$ & $0.4903 /(64) ; 0.5376 /(11) ; 1.4340 /(61)$ & 35.16 & -0.0068 & 84.37 & 0.9810 \\
\hline SOS & $14-58-61-69-70$ & $1.4428 /(61) ; 0.5398 /(11) ; 0.5304 /(65)$ & 35.1624 & 0.0000 & 84.37 & 0.9690 \\
\hline QOCSOS & $14-58-61-69-70$ & $0.5376 /(11) ; 1.4340 /(61) ; 0.4903 /(64)$ & 35.1624 & & 84.37 & 0.9813 \\
\hline
\end{tabular}

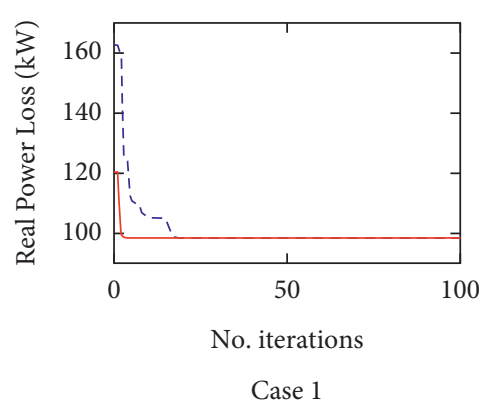

-- SOS
— QOCSOS

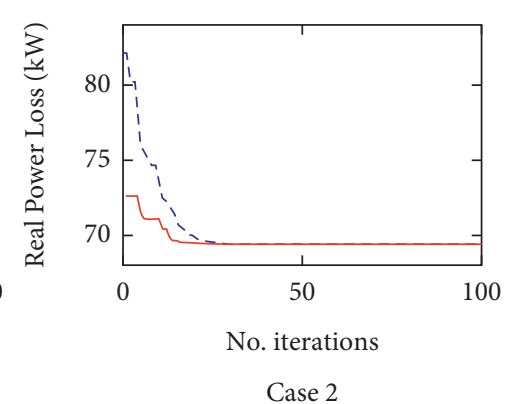

$---\operatorname{SOS}$

Q QOCSOS

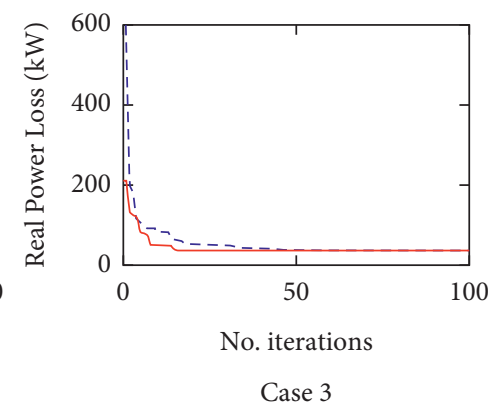

$---\operatorname{SOS}$

- QOCSOS

FIgURE 5: Convergence curves by QOCSOS and SOS for all cases of 69-bus network. (a) Case 1. (b) Case 2. (c) Case 3.

which is better than those of SOS, SFS [16], and ACSA [19]. The QOCSOS method can improve the result $1.0250 \%$, $0.7155 \%, 0.3702 \%$, and $3.6128 \%$ compared to SOS, SFS [16], and ACSA [19], respectively.

The real power losses produced by QOCSOS in Cases 1-3 were $854.0309 \mathrm{~kW}$ (34.21\% PLR), 667.2940 kW (48.59\% PLR), and $565.0605 \mathrm{~kW}(56.47 \%$ PLR), according to Tables $8-10$. It is shown that Case 3 had the greatest PLR value. This claimed that when the ODGP and DNR problems were examined together, the system's real power loss was significantly decreased. In terms of convergence rate, QOCSOS converged to the near-optimal solution at a quicker pace than SOS, as portrayed in Figure 7. The 119-bus network's voltage profiles are shown in Figure 8 for all cases. The minimal voltage values produced by QOCSOS after solving the DNR-DG issue were 0.9323 p.u., 0.9541 p.u., and 0.9599 p.u. for Cases 1-3, respectively. In addition, Case 3 substantially enhanced the voltage profile after simultaneously considering the ODGP and DNR problems, as seen in Figure 8. 


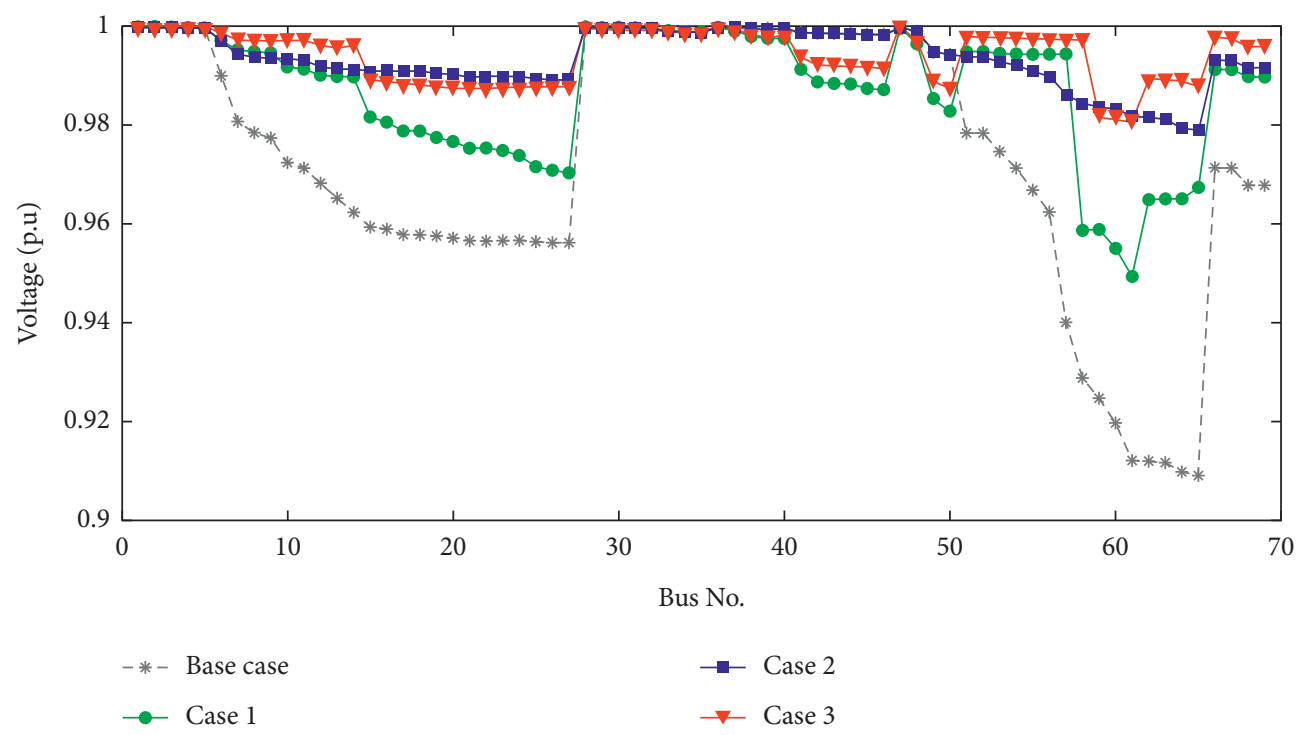

FIgURE 6: Voltage profile for all cases for 69-bus network.

TABLE 8: Optimal configuration obtained by QOCSOS and other techniques for Case 1 of the 119-bus RDN.

\begin{tabular}{|c|c|c|c|c|c|}
\hline Method & Opened switches & $P_{L}(\mathrm{~kW})$ & $R I(\%)$ & PLR (\%) & $V_{\min }$ (p.u.) \\
\hline Base case & $118-119-120-121-122-123-124-125-126-127-128-129-130-131-132$ & 1298.09 & & - & 0.8688 \\
\hline ITS [33] & $42-26-23-51-122-58-39-95-74-71-97-129-130-109-34$ & 867.4 & +1.5413 & 33.18 & 0.9323 \\
\hline MTS [34] & $42-26-23-51-122-58-39-95-74-71-97-129-130-109-34$ & 867.4 & +1.5413 & 33.18 & 0.9323 \\
\hline HSA [31] & $23-27-33-43-53-62-72-75-123-125-126-129-130-131-132$ & 854.21 & +0.0210 & 34.19 & 0.9323 \\
\hline ACSA [19] & $42-25-23-121-50-58-39-95-71-74-97-129-130-109-34$ & 854.06 & +0.0034 & 34.21 & 0.9298 \\
\hline FWA [14] & $42-25-23-121-50-58-39-95-71-74-97-129-130-109-34$ & 854.06 & +0.0034 & 33.18 & 0.9298 \\
\hline SFS [16] & $42-25-23-121-50-58-39-95-71-74-97-129-130-109-34$ & 854.04 & +0.0011 & 34.21 & 0.932 \\
\hline SOS & $23-25-34-39-42-50-59-71-74-95-97-109-128-129-130$ & 857.1404 & +0.3628 & 33.97 & 0.9323 \\
\hline QOCSOS & $23-25-34-39-42-50-58-71-74-95-97-109-121-129-130$ & 854.0309 & & 34.21 & 0.9323 \\
\hline
\end{tabular}

TABLE 9: Optimal DG placement results obtained by QOCSOS and other techniques for Case 2 of 119-bus RDN.

\begin{tabular}{llcccc}
\hline Method & \multicolumn{1}{c}{$P_{D G}(\mathrm{MW}) /(\mathrm{bus})$} & $P_{L}(\mathrm{~kW})$ & $R I(\%)$ & $P L R(\%)$ & $V_{\mathrm{min}}(\mathrm{p} . \mathrm{u})$. \\
\hline SFS [16] & $2.9786 /(71) ; 3.1199 /(109) ; 2.8833 /(50)$ & 667.29 & -0.0006 & 48.59 \\
SOS & $2.8833 /(50) ; 2.9785 /(71) ; 3.1199 /(109)$ & 667.2940 & 0.0000 & 48.59 & 0.9541 \\
QOCSOS & $2.8833 /(50) ; 2.9785 /(71) ; 3.1199 /(109)$ & 667.2940 & & 48.59 & 0.9541 \\
\hline
\end{tabular}

TABLE 10: Optimal DRN-DG problem results obtained by QOCSOS and other techniques for Case 3 of 119-bus RDN.

\begin{tabular}{|c|c|c|c|c|c|c|}
\hline Method & Opened switches & $P_{D G}(\mathrm{MW}) /$ (bus) & $P_{L}(\mathrm{~kW})$ & $R I(\%)$ & PLR (\%) & $V_{\min }$ (p.u.) \\
\hline ACSA [19] & $\begin{array}{c}42-25-22-121-122-58-39-125-70-127-128-81- \\
130-131-33\end{array}$ & $\begin{array}{c}2.5331(50) ; 3.6819(109) ; 3.7043 \\
(73)\end{array}$ & 586.24 & +3.6128 & 53.97 & 0.9644 \\
\hline SFS [16] & $\begin{array}{l}43-16-23-42-50-61-124-125-70-74-75-82-130- \\
108-34\end{array}$ & $\begin{array}{c}2.3133 /(110) ; 4.0392 /(91) \\
8.7132 /(29)\end{array}$ & 567.16 & +0.3702 & 56.31 & 0.9595 \\
\hline SOS & $\begin{array}{c}42-25-22-121-122-58-39-125-70-127-74-129- \\
130-108-33-42\end{array}$ & $\begin{array}{c}2.6517 /(50) ; 4.0922 /(91) \\
2.3055 /(110)\end{array}$ & 570.9123 & +1.0250 & 56.02 & 0.9591 \\
\hline QOCSOS & $\begin{array}{c}23-26-34-39-42-50-61-70-74-85-86-107-121- \\
125-129\end{array}$ & $\begin{array}{c}9.3909 /(4) ; 4.1078 /(91) \\
2.5599 /(110)\end{array}$ & 565.0605 & & 56.47 & 0.9599 \\
\hline
\end{tabular}




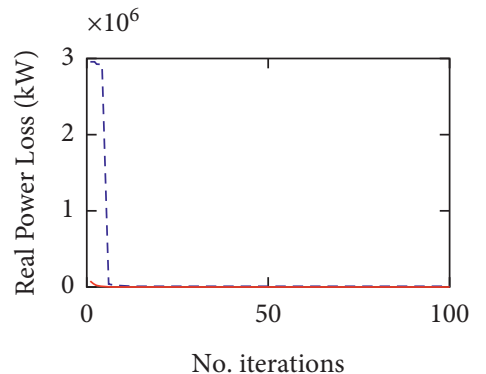

Case 1

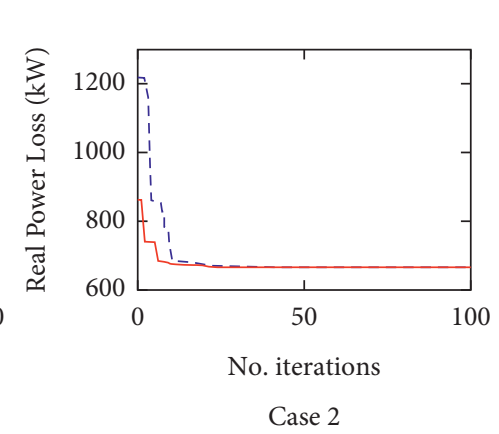

- - SOS

- QOCSOS

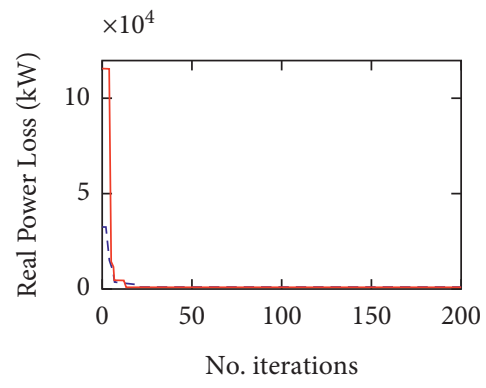

Case 3
- - - SOS

— QOCSOS ase 2

- - SOS

— QOCSOS

Figure 7: Convergence curves by QOCSOS and SOS for all cases of 119-bus network. (a) Case 1. (b) Case 2. (c) Case 3.

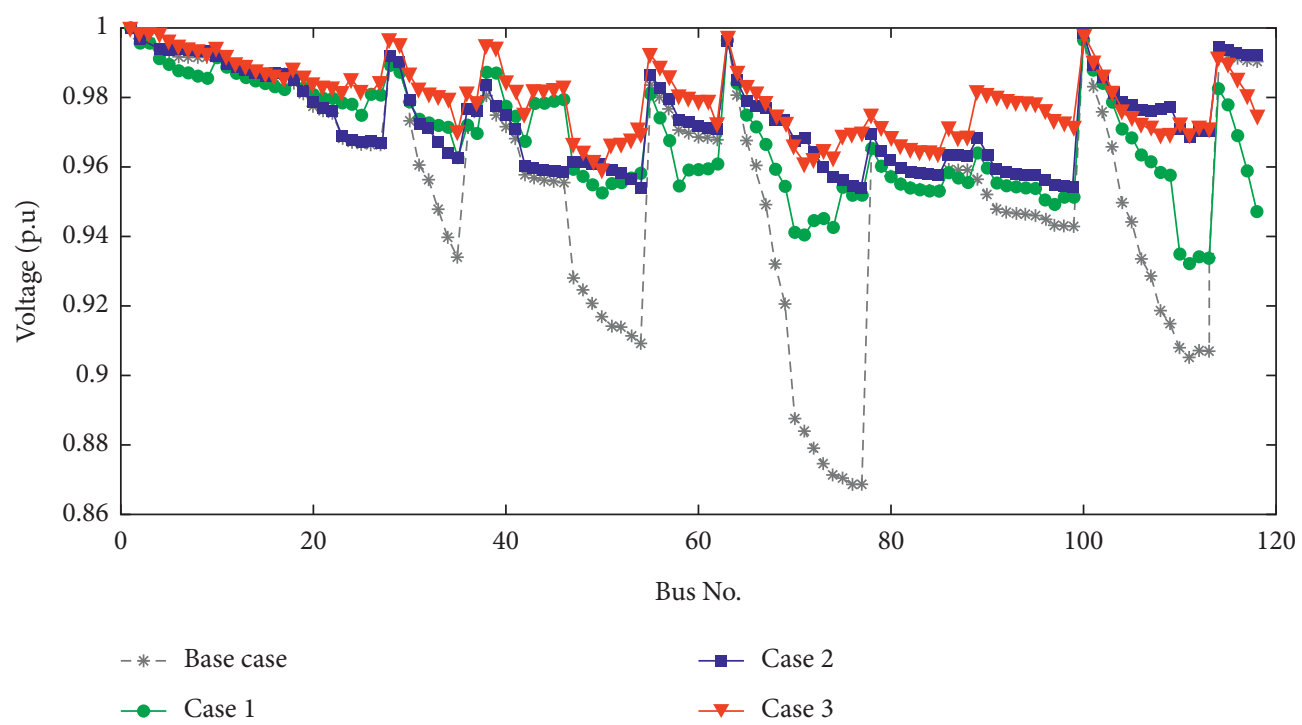

FIGURE 8: Voltage profile for all cases for 119-bus network.

In this case, QOCSOS outperformed the comparable techniques with regard to the solution quality, demonstrating its suitability for a large-scale system.

\section{Conclusion}

In the study, the improved QOCSOS is successfully implemented to solve the simultaneous problems of network reconfiguration and DG allocation in RDNs to reduce the real power loss. The efficacy of QOCSOS has been carried out on the 33-bus, 69-bus, and 119-bus RDNs. It was found that Case 3 (combination of optimal network reconfiguration and DG allocation) offered the best real power loss and minimum voltage amplitude compared to Case 1 (only network reconfiguration) or Case 2 (only optimal DG placement). For this case, the power loss reductions were $74.57 \%, 84.37 \%$, and $56.47 \%$ for the 33-bus, 69-bus, and 119-bus RDNs. Furthermore, the findings showed that the suggested QOCSOS algorithm delivered higher solution quality with regard to loss minimization than the original SOS and several other optimization approaches, particularly for large-scale systems, as seen from the outcome evaluations. As a result, the QOCSOS algorithm provides a viable solution for the DNR-DG problem in RDNs.

\section{Data Availability}

No data were used to support this study.

\section{Conflicts of Interest}

The authors declare that there are no conflicts of interest regarding the publication of this paper.

\section{Acknowledgments}

This research was funded by the Ho Chi Minh City University of Technology, VNU-HCM (Grant no. T-ĐĐT2020-31). 


\section{References}

[1] S.-E. Razavi, E. Rahimi, M. S. Javadi et al., "Impact of distributed generation on protection and voltage regulation of distribution systems: a review," Renewable and Sustainable Energy Reviews, vol. 105, pp. 157-167, 2019.

[2] R. S. Rao, K. Ravindra, K. Satish, and S. V. L. Narasimham, "Power loss minimization in distribution system using network reconfiguration in the presence of distributed generation," IEEE Transactions on Power Systems, vol. 28, no. 1, pp. 317-325, 2013.

[3] A. Merlin and H. Back, "Search for a minimal-loss operating spanning tree configuration in an urban power distribution system," in Proceedings of the 5th Power System Computation Conference (PSCC), Cambridge, UK, September 1975.

[4] S. Civanlar, J. J. Grainger, H. Yin, and S. S. H. Lee, "Distribution feeder reconfiguration for loss reduction," IEEE Transactions on Power Delivery, vol. 3, no. 3, pp. 1217-1223, 1988.

[5] J. A. Martín and A. J. Gil, "A new heuristic approach for distribution systems loss reduction," Electric Power Systems Research, vol. 78, no. 11, pp. 1953-1958, 2008.

[6] V. N. Gohokar, M. K. Khedkar, and G. M. Dhole, "Formulation of distribution reconfiguration problem using network topology: a generalized approach," Electric Power Systems Research, vol. 69, no. 2, pp. 304-310, 2004.

[7] J. Z. Zhu, "Optimal reconfiguration of electrical distribution network using the refined genetic algorithm," Electric Power Systems Research, vol. 62, no. 1, pp. 37-42, 2002.

[8] J. Torres, J. L. Guardado, F. Rivas-Dávalos, S. Maximov, and E. Melgoza, "A genetic algorithm based on the edge window decoder technique to optimize power distribution systems reconfiguration," International Journal of Electrical Power \& Energy Systems, vol. 45, no. 1, pp. 28-34, 2013.

[9] N. Gupta, A. Swarnkar, and K. R. Niazi, "Reconfiguration of distribution systems for real power loss minimization using adaptive particle swarm optimization," Electric Power Components and Systems, vol. 39, no. 4, pp. 317-330, 2011.

[10] L. Li and C. Xuefeng, "Distribution network reconfiguration based on niche binary particle swarm optimization algorithm," Energy Procedia, vol. 17, pp. 178-182, 2012.

[11] R. Pegado, Z. Ñaupari, Y. Molina, and C. Castillo, "Radial distribution network reconfiguration for power losses reduction based on improved selective BPSO," Electric Power Systems Research, vol. 169, pp. 206-213, 2019.

[12] C.-T. Su, C.-F. Chang, and J.-P. Chiou, "Distribution network reconfiguration for loss reduction by ant colony search algorithm," Electric Power Systems Research, vol. 75, no. 2, pp. 190-199, 2005.

[13] A. R. Abbasi, "Investigation of simultaneous effect of demand response and load uncertainty on distribution feeder reconfiguration," IET Generation, Transmission \& Distribution, vol. 14, no. 8, pp. 1438-1449, 2020.

[14] A. Mohamed Imran and M. Kowsalya, "A new power system reconfiguration scheme for power loss minimization and voltage profile enhancement using Fireworks Algorithm," International Journal of Electrical Power \& Energy Systems, vol. 62, pp. 312-322, 2014.

[15] J. Olamaei, T. Niknam, and S. Badali Arefi, "Distribution feeder reconfiguration for loss minimization based on modified honey bee mating optimization algorithm," Energy Procedia, vol. 14, pp. 304-311, 2012.

[16] T. T. Tran, K. H. Truong, and D. N. Vo, "Stochastic fractal search algorithm for reconfiguration of distribution networks with distributed generations," Ain Shams Engineering Journal, vol. 11, no. 2, pp. 389-407, 2020.

[17] S. Teimourzadeh and K. Zare, "Application of binary group search optimization to distribution network reconfiguration," International Journal of Electrical Power \& Energy Systems, vol. 62, pp. 461-468, 2014.

[18] A. Mohamed Imran, M. Kowsalya, and D. P. Kothari, "A novel integration technique for optimal network reconfiguration and distributed generation placement in power distribution networks," International Journal of Electrical Power \& Energy Systems, vol. 63, pp. 461-472, 2014.

[19] T. T. Nguyen, A. V. Truong, and T. A. Phung, "A novel method based on adaptive cuckoo search for optimal network reconfiguration and distributed generation allocation in distribution network," International Journal of Electrical Power \& Energy Systems, vol. 78, pp. 801-815, 2016.

[20] A. Onlam, D. Yodphet, R. Chatthaworn, C. Surawanitkun, A. Siritaratiwat, and P. Khunkitti, "Power loss minimization and voltage stability improvement in electrical distribution system via network reconfiguration and distributed generation placement using novel adaptive shuffled frogs leaping algorithm," Energies, vol. 12, no. 3, 2019.

[21] K. S. Sambaiah and T. Jayabarathi, "Optimal reconfiguration and renewable distributed generation allocation in electric distribution systems," International Journal of Ambient Energy, vol. 42, no. 9, pp. 1018-1031, 2021.

[22] K. H. Truong, P. Nallagownden, Z. Baharudin, and D. N. Vo, "A Quasi-Oppositional-Chaotic Symbiotic Organisms Search algorithm for global optimization problems," Applied Soft Computing, vol. 77, pp. 567-583, 2019.

[23] A. Y. Abdelaziz, F. M. Mohammed, S. F. Mekhamer, and M. A. L. Badr, "Distribution Systems Reconfiguration using a modified particle swarm optimization algorithm," Electric Power Systems Research, vol. 79, no. 11, pp. 1521-1530, 2009.

[24] M.-Y. Cheng and D. Prayogo, "Symbiotic Organisms Search: a new metaheuristic optimization algorithm," Computers \& Structures, vol. 139, pp. 98-112, 2014.

[25] H. R. Tizhoosh, "Opposition-based learning: a new scheme for machine intelligence," in Proceedings of the International Conference on Computational Intelligence for Modelling, Control and Automation and International Conference on Intelligent Agents, Web Technologies and Internet Commerce (CIMCA-IAWTIC'06), vol. 1, pp. 695-701, Vienna, Austria, November 2005.

[26] S. Rahnamayan, H. R. Tizhoosh, and M. M. A. Salama, "Quasi-oppositional differential evolution," in Proceedings of the 2007 IEEE Congress on Evolutionary Computation, pp. 2229-2236, Singapore, September 2007.

[27] J. Ji, S. Gao, S. Wang, Y. Tang, H. Yu, and Y. Todo, "Selfadaptive gravitational search algorithm with a modified chaotic local search," IEEE Access, vol. 5, Article ID 17881, 2017.

[28] D. Jia, G. Zheng, and M. K. Khan, "An effective memetic differential evolution algorithm based on chaotic local search," Information Sciences, vol. 181, no. 15, pp. 3175-3187, 2011.

[29] T. T. The, S. N. Quoc, and D. V. Ngoc, "Symbiotic organism search algorithm for power loss minimization in radial distribution systems by network reconfiguration and distributed generation placement," Mathematical Problems in Engineering, vol. 2020, Article ID 1615792, 2020.

[30] M. E. Baran and F. F. Wu, "Network reconfiguration in distribution systems for loss reduction and load balancing," IEEE Transactions on Power Delivery, vol. 4, no. 2, pp. 1401-1407, 1989. 
[31] R. Srinivasa Rao, S. V. L. Narasimham, M. R. Raju, and A. S. Rao, "Optimal network reconfiguration of large-scale distribution system using harmony search algorithm," IEEE Transactions on Power Systems, vol. 26, no. 3, pp. 1080-1088, 2011.

[32] H.-D. Chiang and R. Jean-Jumeau, "Optimal network reconfigurations in distribution systems. I. A new formulation and a solution methodology," IEEE Transactions on Power Delivery, vol. 5, no. 4, pp. 1902-1909, 1990.

[33] D. Zhang, Z. Fu, and L. Zhang, “An improved TS algorithm for loss-minimum reconfiguration in large-scale distribution systems," Electric Power Systems Research, vol. 77, no. 5, pp. 685-694, 2007.

[34] A. Y. Abdelaziz, F. M. Mohamed, S. F. Mekhamer, and M. A. L. Badr, "Distribution system reconfiguration using a modified Tabu Search algorithm," Electric Power Systems Research, vol. 80, no. 8, pp. 943-953, 2010. 